\title{
Cache optimization cloud scheduling (COCS) algorithm based on last level caches
}

\author{
K. Vinod Kumar, Ranvijay
}

Department of Computer Science and Engineering, Motilal Nehru National Institute of Technology, India

\begin{tabular}{ll}
\hline \hline Article Info & ABSTRACT \\
\cline { 2 - 3 } Article history: & $\begin{array}{l}\text { Recently, the utilization of cloud services like storage, various software, } \\
\text { networking resources has extremely enhanced due to widespread demand of } \\
\text { these cloud services all over the world. On the other hand, it requires huge } \\
\text { Received Apr 23, } 2019 \\
\text { Revised Jun 18,2019 } \\
\text { Accepted Jul 3, 2019 }\end{array}$ \\
$\begin{array}{l}\text { increasing demand. The high demand of these cloud services can lead to high } \\
\text { amount of energy consumption in these cloud centers. Therefore, to eliminate } \\
\text { these drawbacks and improve energy consumption and storage enhancement } \\
\text { Keywords: }\end{array}$ & $\begin{array}{l}\text { Optimization Cloud Scheduling (COCS) Algorithm Based on Last Level } \\
\text { Caches to ensure high cache memory Optimization and to enhance the } \\
\text { processing speed of I/O subsystem in a cloud computing environment which } \\
\text { rely upon Dynamic Voltage and Frequency Scaling (DVFS). The proposed } \\
\text { Dynamic voltage and } \\
\text { frequency scaling }\end{array}$ COCS technique helps to reduce last level cache failures and the latencies of \\
Energy consumption & average memory in cloud computing multi-processor devices. This proposed \\
Last level cache & COCS technique provides an efficient mathematical modelling to minimize \\
energy consumption. We have tested our experiment on Cybershake \\
scientific dataset and the experimental results are compared with different \\
conventional techniques in terms of time taken to accomplish task, power \\
consumed in the VMs and average power required to handle tasks.
\end{tabular}

Copyright (C) 2019 Institute of Advanced Engineering and Science. All rights reserved.

\section{Corresponding Author:}

K. Vinod Kumar, Department of Computer Science and Engineering, Motilal Nehru National Institute of Technology, Allahabad, 211004-India.

Email: vinodkgpt@gmail.com

\section{INTRODUCTION}

In the field of next upcoming generation of computing platform, the application of big data is most emerging application because there is a huge increment in the creation of data and the storage space. As per the research done in 2012, the ever-lasting increment of data has converted a particular dataset of few terabytes data to the several petabytes [1]. The several features like large capacity, bulky distributed datasets and high velocity is mainly considered in the applications of Big Data which requires the diverse processing schemes such that optimization strategies and an accurate decision making can be enabled [2]. In actual world a huge amount of data is generated in many fields like medical, surfing on internet, telecommunication, pharmaceutical, technology of information and the business.

Moreover, in recent time, cloud computing applications has taken immense rise in real world due to its various facilities provided by the cloud providers like 'pay-as-you-go' scheme, massive promotions, easy to use, large connectivity with massive number of subscribers. An application of the cloud computing is distributed type application which allows the user to provide the services as per there demand through the internet [3]. The main reason for the drastic growth of cloud computing is because its application saves huge amount of computational time and capacity required for storing the data and also for accessing the several resources. The different resources which are provided by the cloud providers (i.e. amazon, Microsoft etc.) are 
available in the form of VMs (virtual machines) within Infrastructure-as-a-Service (IaaS) model [4]. In a cloud computing environment, the time required to execute any of the operations on the virtual machines is clearly relied upon the number of instructions handled by $V M$ which is in some millions and processing power which is in million instructions per second per core. However, the execution time is also dependent on the criticality levels of various functions as it can be varied for every function. Thus, an efficient scheduling is highly appreciated to execute millions of instructions at a time in a cloud environment. Therefore, an efficient task scheduling can be utilized to reduce the processing time in numerous functions of cloud computing environment.

Furthermore, various adaptive control methods like dynamic voltage and frequency scaling (DVFS), dynamic speed scaling (DSS) and dynamic frequency scaling $(D F S)$ are introduced by different researchers in recent time to conserve energy and shield environment. Dynamic voltage and frequency scaling (DVFS) is one of the most promising technology which can adaptively optimize power by scaling frequency and voltage. Other energy optimization technologies which are available in the market are like DFS, DSS and $D S P$. All these techniques provide energy-conserving scheduling by diminishing the supply frequency and voltage of the cloud computing environment when numerous jobs are processing adaptively [5-8]. Currently, various chip manufacturing companies provide built-in-processors in integration with DVFS technologies to speed up performance of their network and reduce power consumption in various cloud computing systems like Intel utilizes Intel SpeedStep processor [9], $A R M$ utilizes intelligent energy manager [10] and $A M D$ company uses Power Now processors. The processors which works on higher frequencies can provide decent performance. However, at the same time, they consume immense amount of energy in cloud computing environment. Thus, to ensure high performance and lower energy consumption, DVFS is the most suitable technique currently. The energy can be conserved in the cloud computing devices in two ways such as by scaling voltage and frequency either at task slack period or while processing external peripherals. Therefore, a huge amount of energy can be conserved using DVFS technologies.

Thus, numerous researchers are concerned about the lack of energy conservation in cloud computing environment and hence numerous power efficient technologies are introduced by different researchers to protect environment by enormous amount of power dissipation and enhance the performance of the environment. However, these power efficient technologies require enormous amount of interaction cost between inter-processors. Moreover, these technologies provide insufficient results and energy consumption of cloud computing devices cannot be reduced more due to enormous amount of memory utilization. Furthermore, cache memory optimization is an essential factor to reduce further energy consumption in the cloud computing environment. In recent years, the speed of cloud computing processors and density of main memory has taken immense growth as well as the utilization of I/O sub-systems has extremely enhanced. Specifically, the growth of I/O subsystems in the applications like multimedia and networking has further enhanced the demand of storage capacity. Even though the processing speed of I/O memory sub-systems is highly enhanced, it cannot fulfill the demand of computer sub-systems. Therefore, storage sub-systems are the one of the performances limiting factor and even can be a reason for high energy consumption in cloud computing environment. Therefore, to reduce energy consumption and enhance performance of I/O subsystems in cloud computing environment, the optimization of cache is an essential factor.

Furthermore, the storage devices in which cache memory is utilized are termed as fast storage subsystems. However, the capacity of these fast storage sub-systems is limited and hence, replace methods should be utilized to enhance the efficiency of storage devices and cache memory. Moreover, some of the well-known cache replace algorithms utilizes FIFO,LFU and $L R U$ and their relevant technologies. The cache hit ratio is utilized to enhance the performance of cloud computing environment which rely upon the information reference confinedity. The performance of I/O subsystem is clearly depending on the processing speed of storage sub-systems which need to be similar in all the storage devices. However, this often does not happen as the processing speed of storage devices varies system to system. Therefore, there is a need of a technique, which maintains the cache hit ratio and enhance the accessing speed of the network by optimizing cache memory. Moreover, energy consumption and higher memory utilization can be minimized by reducing the traffic on shared cache memories and memory handlers. The additional energy consumption and slower processing in the cloud computing environment is due to missing of last level caches $(L L C)$ on shared storage sub-systems.

Therefore, these problems need to be focused soon so that the performance and memory capacity of cloud computing devices get enhanced. Thus, here, we have adopted a Cache Optimization Cloud Scheduling (COCS) Algorithm Based on Last Level Caches to ensure high cache memory Optimization and to enhance the processing speed of $\mathrm{I} / \mathrm{O}$ subsystem in a cloud computing environment which rely upon Dynamic Voltage and Frequency Scaling $(D V F S)$. The proposed cache Optimization technique helps to minimize the mismanagement of last level caches and to identify the behaviors of cache patterns. This technique decreases congestion on shared cache memories and relocates memory sub-systems dynamically. The memory capacity

Cache optimization cloud scheduling (COCS) algorithm based on last level caches (K. Vinod Kumar) 
of cloud computing environment can be enhanced by sharing caches between cores dynamically. The experimental outcomes verify that the proposed Cache Optimization Cloud Scheduling (COCS) Algorithm can provide higher performance in terms of energy consumption, memory capacity and efficiency in cloud computing environment even in the worst-case scenarios.

This paper is presented in following sections which are as follows. In section 2, we describe about the related work to cache memory and their importance and drawbacks in existing techniques and in section 3, we described our proposed Cache Optimization Cloud Scheduling (COCS) methodology. In section 4, experimental results and evaluation shown and section 5 concludes our paper.

\section{RELATED WORK}

In recent years, the concerns related to enormous memory utilization and performance in cloud computing environment have taken immense growth. Thus, performance enhancement and proper management of storage sub-systems is highly critical need. Moreover, to enhance the storage in cloud computing environment, the optimization of cache memory. Therefore, a widespread literature survey is introduced on proper utilization of storage sub-systems and energy aware scheduling algorithms and their link with DVFS in a multi-core heterogeneous cloud computing environment.

In [11], an algorithm for the efficient management of shared caches and their effective partitioning is presented to reduce the accessing of main memory in cloud computing environment. This technique helps to minimize the arithmetic and addressing operations. The architecture of last level cache is introduced to reduce loop tiling problems. However, the balancing of energy consumption and performance is a challenging task. In [12], a cache energy Optimization technique is introduced by interchanging from high speed LI cache to L1 low speed cache using the DVFS application. This model provides a cache hierarchy to model a low-power cache algorithm. This method helps to reduce congestion and also decrease extra latency of main memory. However, a proper cache switching modelling is required to utilize this technique in real time. In [13], an efficient cache architecture is presented for DVFS-enabled devices top reduce the cache overhead in the cloud computing environment. This technique is capable of handling faults by changing associativity adaptively in the network. This technique also helps to eliminate redundant information present in the network. In [14], an efficient caching technique is introduced to compare performance of the network with various state-of-art-techniques. The static cache and redis cache technique help to reduce congestion in the cloud computing network and to provide proper resource utilization. In [15], an accurate memory frequency scaling strategy is introduced using Graphics Processing Units (GPUS) based on dynamic voltage and frequency scaling (DVFS) technique to reduce energy consumption and enhance performance of the network. This strategy optimizes L2 cache, shared memory and L1 cache memory. In [16], various cache bypassing methods are introduced based on CPU and GPU cores and compared with each other. This technique helps to enhance the capacity of cache memory and decrease energy consumption in larger caches. However, the problems like high congestion in the network and miss rate can be enhanced while using cache by-passing techniques. In [17], a cache by-passing technique is presented for various mobile SOCs which are clock domain and DVFS enabled. This technique helps to enhance performance of the network and energy conservation in DVFS-enabled CPUs. Here, last level caches are directly accessed to enhance energy conservation and performance of the system. In [18], a novel cache Optimization technique is presented based on Dynamic voltage and frequency scaling to enhance the reliability of the cloud computing network. This technique helps to enhance performance and capacity of the system. However, overhead of the network is very high using this technique.

Various researchers have introduced different cache memory Optimization techniques in above literatures. However, very few methods can be utilized in real-time due to various problems like high overhead, high energy consumption, slower performance and unable to reduce cache memory $[11,12,14,17$ 19]. Thus, we have adopted a Cache Optimization Cloud Scheduling (COCS) Algorithm Based on Last Level Caches to ensure high cache memory Optimization and to enhance the processing speed of I/O subsystem in a cloud computing environment based on Dynamic voltage and Frequency Scaling (DVFS) technique.

\section{PROPOSED CACHE OPTIMIZATION CLOUD SCHEDULING (COCS) ALGORITHM MODELLING}

This section provides detailed modelling for the proposed Cache Optimization Cloud Scheduling (COCS) technique. Here, we introduce a Cache Optimization Cloud Scheduling (COCS) Algorithm Based on Last Level Caches to ensure high cache memory Optimization and to enhance the processing speed of I/O subsystem in a cloud computing environment based on Dynamic voltage and Frequency Scaling (DVFS) technique. The architecture of the proposed Cache Optimization Cloud Scheduling (COCS) technique is 
presented in Figure 1. Here, we provide efficient modeling to reduce energy consumption and enhance capacity and efficiency of the cloud computing network. The proposed cache Optimization technique migrates cloud virtual machines to reduce the final Last Level Cache $(L L C)$ failures in the cloud computing network. This technique contains confined and generalized scheduling stages. Here, Figure 1 demonstrates the proposed cache Optimization technique which identifies the behavior of various caches in every $V M$ from different working nodes and gathers all the VMs to decrease the final cache failures and the latencies of average memory in the cloud computing network. Figure 1 defines the complete architecture of the proposed COCS model. In each working node, $L L C$ failures are checked to measure the performance of the system. The monitoring system measures the $L L C$ failures per VM and transfer it to the cloud computing scheduler. The generalized scheduling is depending on the status of VMs from each node. The proposed COCS Algorithm utilizes the measured information of cloud computing VMs. Initially, the COCS technique locates VMs on working nodes which takes CPU and memory for every node to enhance the behavior of cache memories.

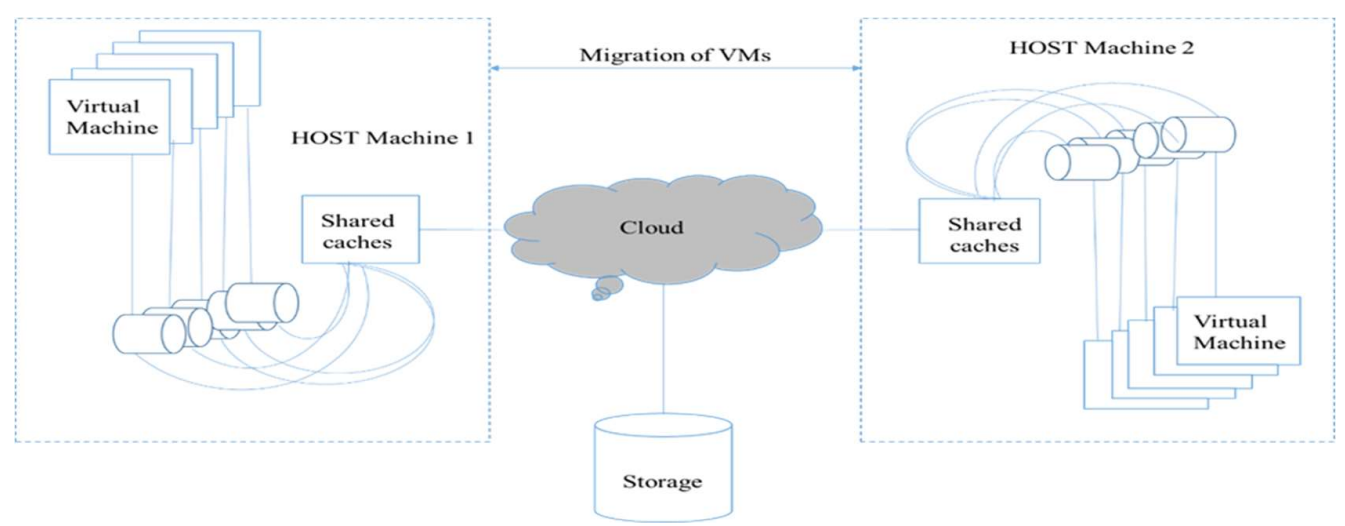

Figure 1. Architecture diagram of proposed Cache Optimization Cloud Scheduling (COCS) model

\subsection{Modeling of cache-aware cloud scheduling}

This section provides a detailed modeling for proposed cache-aware cloud scheduling technique. Initially, the proposed cache Optimization techniques takes conflicts of shared cache memories. Then, migrates cloud virtual machines to reduce the final Last Level Cache $(L L C)$ failures in the cloud computing network. This technique contains confined and generalized scheduling stages. All the VMs are gathered together to at each working node and planned to domains of mutual caches. The optimization of VMs is presented to enhance the network bandwidth and network capacity. The cloud simulator scheduler is utilized to reallocate VMs at the nodes where $L L C$ failures in a computing network of generalized stage. Here, Algorithm 1 is demonstrated in Table 1 which describes the proposed COCS Algorithm based on Last level caches. This cache aware scheduling algorithm distributed into two stages like one is confined stage and other one is generalized stage. All the VMs at every working nodes are classified by LLC failures and then gathered together according to their missed last level caches in the domains of shared caches. The virtual machines with the most LLC failures fit in group 1, next VM with most $L L C$ in group 2. Similarly, the virtual machines with the least LLC failures fits in group 1 and next VM with least $L L C$ in group 2. This scheme helps to allocate all the VMs in either of the group.

The cloud scheduler realizes two types of nodes present in the cloud computing network such as node with highest $L L C$ failures and node with least $L L C$ failures in the generalized phase. If the difference between $L L C$ failures is more than threshold value then both the VMs are swapped using cache optimization technique. The cloud scheduler performs two-stage scheduling progressively by decreasing the final $L L C$ failures in a cloud computing network after a regular interval of time.

Algorithm 1 Cache Optimization Cloud Scheduling (COCS)Algorithm Step 1: N_L $=<\mathrm{n} \_\mathrm{x} 1, \ldots, \mathrm{n} \_\mathrm{x} 2>/ /$ LLC miss of every computing node Step 2: $\overline{\mathrm{W}}_{-} \mathrm{L}=<\overline{\mathrm{w}} \_\mathrm{x} 1, \ldots, \mathrm{w} \_\mathrm{x} 2>/ /$ LLC miss of VMs in each working nodes $/ /$ confined stage

Step 3: for every working node $j$ from 1 to $y$ do // identify LLC failures in for every VM in working node $j$ Step 4: $n x_{j} \Leftarrow$ colllects $(j)$ 
Step 5: $W_{\mathbb{L}} \Leftarrow \operatorname{sort}\left(n x_{j}\right) / /$ disperse VMs with LLC failures

Step 6: disperse $\left(W_{\mathbb{L}}\right)$

Step 7: end for

// Generalized stage

// identify working nodes with largest and least LLC failures

Step 8: largeNode $\Leftarrow$ search largeNode $\left(N_{\mathbb{L}}\right)$

Step 9: leastNode $\Leftarrow$ search leastNode $\left(N_{\mathbb{L}}\right)$

// search VMs which contains maximum and minimum LLC failures

Step 10: largeVM $\Leftarrow$ search largestVM(largeNode)

Step 11: least $V M \Leftarrow$ search leastVM (leastnode)

Step 12: if $T<\operatorname{largeNode}_{\mathbb{L L C}}-$ leastNode $_{\mathbb{L L C}}$ then

Step 13: interchange (largeVM, leastVM)

Step 14: end if

\subsection{Modelling for minimizing energy consumption using COCS}

In this section efficient mathematical modelling is presented to minimize energy consumption in multicore cloud computing systems using proposed cache optimization cloud scheduling technique. This technique helps to minimize energy consumption in cloud devices using cache minimization concept by eliminating dynamic constrained minimization problem. The energy consumption $P(t)$ occurs in any multicore cloud computing processor is expressed as

$$
P(t)=e(t) M_{a}
$$

Where, $P(t)$ represents the energy consumption in a cloud computing processor in the $t^{\text {th }}$ managing period. Here, $e(t)$ represents power consumption of a cloud computing multi-core processor which is linked with both frequencies of the core $B_{k}$ and size of the current $L 2$ cache which remains a constant for the frequencies of the core $B_{k}$, size of the current L2 cache and task-load of the system and remain unchanged throughout in every managing period whereas $M_{a}$ is managing period to release multiple illustrations of every task during $t^{t h}$ managing period. Here, $v_{k}(t)$ represents the core $\left(B_{k}\right)$ utilization in a $t^{\text {th }}$ managing period which depends on the statistics produced by an operating system. Here, the cloud computing processor consists of two cache levels L1 and L2 which are shared with homogenous cores in multi-core shared architecture. In the proposed model each cloud computing processors support DVFS-enabled cores which conserve high amount of energy. The cache memory is divided into various tasks. The level 2 cache memory divider can be denoted as $a_{k}(t)$ for a core size $B_{k}$. The maximum core frequency for a core size $B_{k}$ can be expressed as $\mathbb{f}_{k_{\uparrow}}(t)$. Then, energy consumption $P(t)$ of the cloud computing multi-core processor can be expressed as

$$
\begin{aligned}
& \min _{a_{k}(t)\left|1 \leq k \leq i, \mathbb{f}_{k}(t)\right| 1 \leq k \leq i} \sum_{k=1}^{i}\left[V_{k}-v_{k}(t)\right]^{2}, \\
& \min _{a_{k}(t)\left|1 \leq k \leq i, \mathbb{f}_{k}(t)\right| 1 \leq k \leq i} P(t) \\
& R_{\downarrow, k} \leq \mathbb{f}_{k}(t) \leq R_{\uparrow, k} \text { where, }(1 \leq k \leq i) \\
& \sum_{k=1}^{i} a_{k}(t) \leq A
\end{aligned}
$$

Where, $V_{k}$ is a Utilization points of sets in which $V=\left[V_{1}, \ldots \ldots, V_{i}\right]^{T}$ for a frequency range of $\left[R_{\uparrow, k}, R_{\downarrow, k}\right]$ for every core $B_{k}$ and overall size of L2 caches cloud computing processors is denoted by $A$ whereas $\left\{a_{k}(t) \mid 1 \leq k \leq i\right\}$ represents the size of cache memory partition and $\left\{\mathbb{f}_{k}(t) \mid 1 \leq k \leq i\right\}$ denotes the frequency of cores in a $t^{t h}$ managing period to reduce the difference between core utilization $v_{k}(t)$ and Utilization points of sets $\left(V_{k}\right)$. Here, the (2) represents the least energy consumption in any cloud computing multicore processor due to constant power generation $e(t)$ for the $t^{t h}$ managing period. Here, the (3) represents shows that the frequency of CPU usually lies in the acceptable range for every core suing the proposed COCS technique. The change in the frequency of any cloud computing device depends on the processors utilized. The (4) represents summation of each divided cache memory which is equivalent to the overall cache memory of the cloud computing processor. For every core of the processor, the difference between core utilization $v_{k}(t)$ and Utilization point of sets $\left(V_{k}\right)$ is minimized using the proposed cache

Int. J. of Adv. in Appl. Sci. Vol. 8, No. 3, September 2019: $184-194$ 
optimization technique by changing size of cache partition and frequency of cores. However, these processes decrease the speed of the cloud computing processors. Therefore, to enhance the speed and performance of the system a dynamic model is introduced to maintain a link between managing $v_{k}(t)$ and manipulated $a_{k}(t)$ factors and core frequency $\mathbb{f}_{k}(t)$ in the $t^{t h}$ managing period. Initially, for a core $B_{k}$, the proposed dynamic model provides a link between $b_{k p}(t)$, task operating time $M_{k p}$ and both manipulated factors $\mathbb{f}_{k}(t)$ in the $t^{t h}$ managing period and $a_{k}(t)$. Then, $b_{k p}(t)$ can be of operates in various manners like frequency dependent and independent segments and can be represented as

$$
b_{k p}(t)=i_{k p} \cdot\left(\mathbb{f}_{k}(t)\right)^{-1}+s_{k p}(t)
$$

Where, $i_{k p} \cdot\left(\mathbb{f}_{k}(t)\right)^{-1}$ is a segment which rely upon frequency whereas $s_{k p}(t)$ represents the a segment which is independent of frequency for an operating time $M_{k p}$ due to operating speed of I/O devices does not rely upon the frequencies of core. These I/O devices does not participate at the time of executing task. Then, the reserved cache memory for a task operating time $M_{k p}$ is denoted as $s_{k p}(t)$ which defines a strong link between size of cache memory and the number of cache failures. Then, a link between $s_{k p}(t)$ and $a_{k p}(t)$ and allocated cache size for a multi-core architecture $B_{k}$ is expressed as

$$
s_{k p}(t)=\left\{\begin{array}{c}
D_{k p} a_{k p}(t)+H_{k p} 0 \leq a_{k p}(t) \leq X_{k p} \\
\text { Constant } a_{k p}(t) \geq X_{k p}
\end{array}\right.
$$

Where, $X_{k p}$ is the size of operating set within a task operating time $M_{k p}$ whereas $D_{k p}$ and $H_{k p}$ are the specified task factors. Here, the (7) describes that using the proposed COCS technique, whenever size of operating set $X_{k p}$ is larger than $a_{k p}(t)$, and then the size of cache memory enhances and can lead to a minimum operating time. Similarly, whenever size of operating set $X_{k p}$ is smaller than $a_{k p}(t)$, then cache failure rate becomes high and cannot be handled by assigning an extra cache memory. Then, to manage realtime task, the link between overall independent frequency and operating time of every task for multi-core processor $B_{k}$ and size of overall cache $a_{k}(t)$ allocated to core $B_{k}$ can be expressed as

$$
s_{k}(t)=\left\{\begin{array}{c}
\sum_{p} D_{k p}{ }^{\prime} a_{k}(t)+\sum_{p} H_{k p} 0 \leq a_{k}(t) \leq X_{k} \\
\text { Constant } a_{k}(t) \geq X_{k}
\end{array}\right.
$$

Where, $D_{k p}{ }^{\prime}=D_{k p} a_{k p}(t) \cdot\left(a_{k}(t)\right)^{-1}$ and $X_{k}=\sum_{p} X_{k p}$. Here, the (8) represents the summation of (7) for each task on a multi-core processor $B_{k}$. Then, the proposed COCS technique helps to minimize interference between shared caches resources of various cores and can be expressed as

$$
h_{k}(t)=\sum_{p} i_{k p} q_{k p} \cdot\left(\mathbb{f}_{k}(t)\right)^{-1}+\sum_{p} D_{k p}{ }^{\prime} q_{k p} a_{k}(t)+\sum_{p} H_{k p} q_{k p}
$$

Where, $h_{k}(t)$ represents the predicted core utilization and $q_{k p}$ represents rate of the task within operating time $M_{k p}$ for a multi-processor core $B_{k}$. From (9), it is verified that $h_{k}(t)$ is inversely proportional to the frequency of core $\mathbb{f}_{k}(t)$. The predicted change in utilization $\Delta h_{k}(t)$ for a multi-core architecture $B_{k}$ can be expressed as

$$
\Delta h_{k}(t)=l_{k}(t) \sum_{p} i_{k p} q_{k p}+\Delta a_{k}(t) \sum_{p} D_{k p}{ }^{\prime} q_{k p}
$$

Where, $l_{k}(t)=\left(\mathbb{f}_{k}(t)\right)^{-1}-\left(\mathbb{f}_{k}(t-1)\right)^{-1}$ and $\Delta a_{k}(t)=a_{k}(t)-a_{k}(t-1)$. Here, $\Delta h_{k}(t)$ can be termed as the linear function of $l_{k}(t)$ and $\Delta a_{k}(t)$. Here, the (10) replaces the direct utilization of frequency of core $\mathbb{f}_{k}(t)$ to $l_{k}(t)$. The (10) shows that $\Delta h_{k}(t)$ is directly proportional to $i_{k p}$ and $D_{k p}{ }^{\prime}$. Then, the cost function of cloud computing system can be reduced with the help of controller for a multi-core $B_{k}$ as

$$
Z_{k}(t)=\sum_{c=1}^{E}\left\|v_{k}(t+c-1 \mid t)-\beta \mathbb{1}_{k}(t+c-1 \mid k)\right\|^{2}+\left\|u_{k}(t \mid t)-u_{k}(t-1 \mid t)\right\|^{2}
$$

Where,

$$
R_{\downarrow, k} \leq \mathbb{f}_{k}(t) \leq R_{\uparrow, k}
$$


Where, $u_{k}(t)=\left[\begin{array}{c}l_{k}(t) \\ \Delta a_{k}(t)\end{array}\right]$ and $E$ represents estimated horizon to estimate the behavior of the device in $E$ managing periods. Here, $\beta \mathbb{f}_{k}(t+1 \mid t)$ represents the trajectory with respect to the utilization factor $v_{k}(t+c-1 \mid t)$ must change from the present utilization factor $v_{k}(t)$ to utilization point of sets $V_{k}$. The size of cache $a_{k}(t)$ for a multi-core system $B_{k}$ is limited by $a_{\text {quota, }}$ to satisfy (5). From this above modelling, the optimization of cache memory can be easily achieved and optimization and least square problems are also minimized. The optimized power consumption can be presented using our proposed COCS technique as

$$
e_{k}(t)=S_{k} \mathbb{f}_{k}(t)^{3}+Y_{k} a_{k}(t)+C_{k}
$$

Where,

$$
\begin{aligned}
& R_{\downarrow, k} \leq \mathbb{f}_{k}(t) \leq R_{\uparrow, k} \\
& a_{k}(t) \leq a_{\text {quota,k }}
\end{aligned}
$$

Where, $S_{k}, Y_{k}$ and $C_{k}$ are the power factors of the cloud computing multi-core processor. The power consumption of cloud computing multi-core processor is represented as the sum of the power consumption of cores and shared caches. The total power consumption is directly depending on the dynamic components of power $S_{k} \mathbb{f}_{k}(t)^{3}$ and leakage power $C_{k}$. Therefore, the power consumption in cache can be optimized using the proposed COCS technique. In this way, energy consumption of a cloud computing model is minimized and performance of the system can be enhanced.

\section{PERFORMANCE EVALUATION}

In this modern era, the utilization of cloud computing embedded devices in daily life has tremendously increased due to abundant utilization of various portable gadgets, information systems and digital appliances etc. However, these embedded devices require high amount of power to operate properly, Thus, the performance of this multi-core cloud computing systems and embedded devices must be very high due to satisfy the ample demand of the market. However, in recent time, the performance of various cloud computing centers has degraded due to large amount of energy consumption in these cloud computing centers. Another major problem while using the cloud computing systems is the storage of the system. The amount of data present in these cloud computing systems is extremely high and requires ample amount of storage to handle these large data. Therefore, to maintain a balancing between high amount of energy consumption and storage enhancement of cloud computing systems, we have introduced a Cache Optimization Cloud Scheduling (COCS) Algorithm Based on Last Level Caches to ensure high cache memory Optimization and to enhance the processing speed of $\mathrm{I} / \mathrm{O}$ subsystem in a cloud computing environment which rely upon Dynamic Voltage and Frequency Scaling (DVFS). The proposed cache Optimization technique helps to minimize the mismanagement of last level caches and to identify the behaviors of cache patterns. Here, we have tested our model on Cybershake scientific dataset and various sizes of tasks are considered like 30,50,100, and 1000 tasks to calculate time taken to accomplish tasks. Cybershake Workflow is produced with the help of four type of jobs like 30,50,100 and 1000. It is used for the Southern California Earthquake center to identify earthquake dangers in a specified place [20]. It requires large amount of storage and CPU resources. The experimental outcomes are presented in terms of time taken to execute task of different size, power consumed in the VMs and average power required to handle tasks against number of tasks considered. Different factors are considered to calculate operating time and energy consumption which is demonstrated in the following section I Table 1. Our proposed COCS model carried out on 64-bit windows 10 OS with 16 GB RAM which contains an INTEL $(R)$ core $(T M) i 5-4460$ processor. It consists of $3.20 \mathrm{GHz}$ CPU. This project is simulated using EclipseWS Neon.3 editor and code is written in $J A V A$.

\subsection{Comparative analysis}

Recently, the modern high-tech cloud computing systems are attempting to match high-end device performance to fulfill the extreme demand of market. However, achieving high-end system performance can head to the extreme energy consumption in these high-tech cloud centers. This is due to this device are mainly battery-oriented and it is very critical to accurately control high amount of energy consumption. If not, then the lifespan of these devices becomes limited due smaller battery life and it may frustrate cloud

Int. J. of Adv. in Appl. Sci. Vol. 8, No. 3, September 2019: $184-194$ 
subscribers. Therefore, efficient task scheduling and storage capacity enhancement by removing unwanted data is the best way to keep up with the high performance and minimum energy consumption. Therefore, here, we have introduced a Cache Optimization Cloud Scheduling (COCS) Algorithm Based on Last Level Caches to ensure high cache memory Optimization and to enhance the processing speed of I/O subsystem in a cloud computing environment which rely upon Dynamic Voltage and Frequency Scaling (DVFS). The proposed technique helps to maintain balancing between high perform and minimum energy consumption. Therefore, here, we have tested our experiment on Cybershake scientific dataset and the experimental results are compared with different conventional techniques in terms of time taken to accomplish task, power consumed in the VMs and average power required to handle tasks. These Cybershake scientific dataset is widely utilized in evaluating the performance of various scheduling techniques and Cybershake workflow is utilized in our experiment. Here, Table 1 demonstrates the performance comparison of our proposed COCS technique in terms of task completion time, power sum, average power and power consumption occurs in VMs using Cybershake scientific dataset of different size like 30, 50,100 and 1000. Power consumption using the proposed COCS technique for Cybershake 30 is 128.4845 Watts, Cybershake 50 is 151.5101 WattsCybershake 100 is 258.672 Watts and Cybershake 1000 is 1696.490 Watts demonstrated in Table 1 which is extremely low in contrast to conventional standard technique. The proposed COCS technique compares Average task completion time using the proposed COCS technique is compared with other conventional techniques utilizing Cybershake scientific dataset of different size like 30, 50,100 and 1000 as demonstrated in Table 1. The Average Task Completion Time with the proposed COCS technique for scientific dataset Cybershake 30 is $0.368431 \mathrm{sec}$, Cybershake 50 is $0.193506 \mathrm{sec}$, Cybershake 100 is $0.140568 \mathrm{sec}$ and Cybershake 1000 is $0.058476 \mathrm{sec}$ and compared with various state-of-art-techniques. The average task completion time is much smaller than any other state-of-art techniques like EMS-C, SPEA2, MODE, NSPSO, $\in-F U Z Z Y$ PSO, MOHEFT [20] using our proposed COCS technique demonstrated in Table 2. The average Power required is much smaller than any other state-of-art techniques like EMS-C, SPEA2, MODE, NSPSO, $\in-F U Z Z Y$ PSO, MOHEFT [20] using our proposed COCS technique demonstrated in Table 2. The VMs types with description used in our experiment is shown at Table 3 . The machine configuration used to compute these results are demonstrated in Table 4. The Power Consumption required is much smaller than any other state-of-art techniques like EMS-C, SPEA2, MODE, NSPSO, $\in$ -FUZZY PSO, MOHEFT [20] using our proposed COCS technique demonstrated in Table 2. The machine configuration used to compute these results are is demonstrated in Table 5. Figure 2 demonstrates the internal architecture of Cybershake workflow.

Table 1. Various parameters comparison for proposed COCS technique vs DVFS using scientific model Cybershake

\begin{tabular}{ccccccc}
\hline \multirow{2}{*}{ Parameters } & & $\begin{array}{c}\text { Total Execution } \\
\text { Time }(\mathrm{s})\end{array}$ & $\begin{array}{c}\text { Power Sum } \\
(\mathrm{W})\end{array}$ & $\begin{array}{c}\text { Average Power } \\
(\mathrm{W})\end{array}$ & $\begin{array}{c}\text { Power Consumption } \\
(W h)\end{array}$ \\
\hline \multirow{6}{*}{ DVFS } & Cybershake 30 & $\mathrm{VM}=20$ & 6359.41 & 12175922.64 & 19.146320 & 3495.42 \\
& Cybershake 50 & $\mathrm{VM}=30$ & 14448.90 & 29068552.89 & 20.118183 & 8518.39 \\
& Cybershake 100 & $\mathrm{VM}=50$ & 30124.41 & 61177338.40 & 20.308229 & 18966.33 \\
& Cybershake 1000 & $\mathrm{VM}=30$ & 74543.57 & 149968122.08 & 20.118184 & 236303.28 \\
& Cybershake 30 & $\mathrm{VM}=20$ & 262.73 & 415469.6734 & 15.8135 & 128.4845 \\
COCS & Cybershake 50 & $\mathrm{VM}=30$ & 283.68 & 451080.8167 & 15.9010 & 151.5101 \\
& Cybershake 100 & $\mathrm{VM}=50$ & 443.21 & 704751.2215 & 15.9010 & 258.672 \\
& Cybershake 1000 & $\mathrm{VM}=30$ & 1328.05 & 2111745.021 & 15.9010 & 1696.490 \\
\hline
\end{tabular}

Table 2. Runtime ratios of the peer algorithms against the proposed EMS-C on the real-world workflows (i.e., runtime (peer algorithm) =runtime (COCS))

\begin{tabular}{lccccccccc}
\hline \multicolumn{1}{c}{ DAGs } & \multirow{2}{*}{$\begin{array}{c}\text { Number } \\
\text { of nodes }\end{array}$} & EMS-C & DVFS & SPEA2 & MODE & NSPSO & $\begin{array}{c}\text { Average Execution time }(\mathrm{s}) \\
\text { PSO }\end{array}$ & MOHFET & COCS \\
\hline Cybershake 30 & 30 & 23.77 & 211.98 & 1.62 & 1.18 & 21.25 & 15.04 & 10.39 & 0.368431 \\
Cybershake 50 & 50 & 29.32 & 288.978 & 1.28 & 1.37 & 30.17 & 26.81 & 38.16 & 0.193506 \\
Cybershake 100 & 100 & 31.53 & 301.244 & 0.92 & 1.31 & 44.75 & 42.22 & 110.42 & 0.140568 \\
Cybershake 1000 & 1000 & 22.71 & 74.54 & 0.32 & 0.97 & 70.72 & 69.56 & -- & 0.058476 \\
\hline
\end{tabular}

Table 3. VMs types with description used in our experiment

\begin{tabular}{cccc}
\hline Type & Memory (GB) & Core Speed (ECU) & Cores \\
\hline m1. small & 1.7 & 1 & 1 \\
m1. large & 7.5 & 4 & 2 \\
m1. xlarge & 15 & 8 & 4 \\
\hline
\end{tabular}




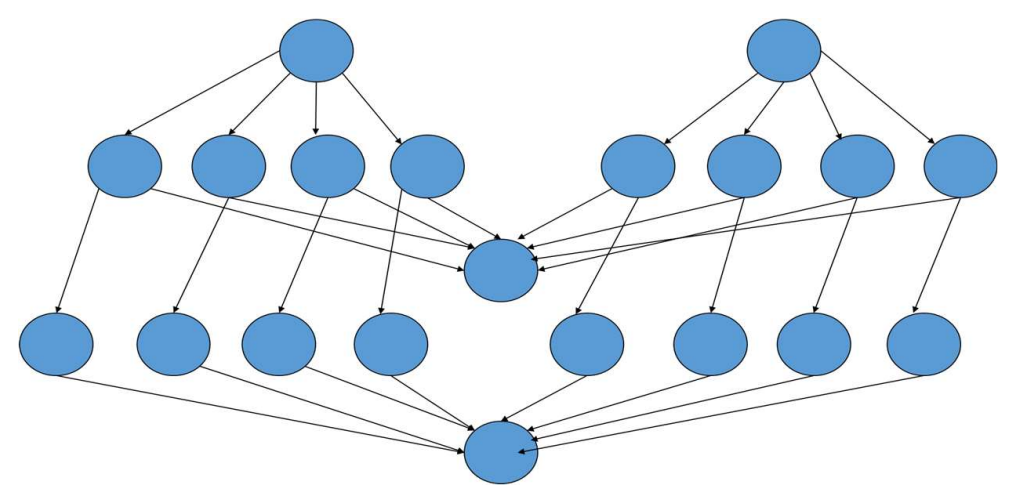

Figure 2. Internal architecture of Cybershake workflow

Table 4. Runtime ratios of the peer algorithms against the proposed EMS-C on the real-world workflows

\begin{tabular}{lcccccccc}
\multicolumn{7}{c}{ (i.e., average power (peer algorithm) } & \multicolumn{7}{c}{ average power (COCS)) } \\
\hline \multicolumn{1}{c}{ DAGs } & $\begin{array}{c}\text { Number } \\
\text { of nodes }\end{array}$ & EMS-C & DVFS & SPEA2 & MODE & NSPSO & $\in$-fuzzy PSO & COCS \\
\hline Cybershake 30 & 30 & 34.0079 & 141.02 & 2.317 & 1.688 & 30.402 & 21.51782 & 0.5271166 \\
Cybershake 50 & 50 & 48.186 & 156.72 & 2.103 & 2.251 & 49.583 & 44.06124 & 0.31802 \\
Cybershake 100 & 100 & 35.666 & 151.92 & 1.040 & 1.481 & 50.621 & 47.75910 & 0.15901 \\
Cybershake 1000 & 1000 & 6.175 & 52.191 & 0.087 & 0.263 & 19.230 & 18.91500 & 0.015901 \\
\hline
\end{tabular}

Table 5. Runtime ratios of the peer algorithms against the proposed EMS-C on the real-world workflows

\begin{tabular}{|c|c|c|c|c|c|c|c|c|}
\hline DAGs & $\begin{array}{l}\text { Number } \\
\text { of nodes }\end{array}$ & \multicolumn{7}{|c|}{ Power consumption } \\
\hline Cybershake 30 & 30 & 276.31 & 1145.8 & 18.83 & 13.71 & 247.02 & 174.8320 & 4.2828166 \\
\hline Cybershake 100 & 100 & 580.21 & 2471.4 & 16.92 & 24.10 & 823.48 & 776.9287 & 2.58672 \\
\hline Cybershake 1000 & 1000 & 658.85 & 5568.3 & 9.283 & 28.14 & 2051.7 & 2018.056 & 1.69649 \\
\hline
\end{tabular}

\subsection{Graphical representation}

This section describes about the graphical demonstration of experimental outcomes using proposed COCS technique. Here, Figure 3 demonstrates task completion time Comparison of proposed COCS technique with conventional DVFS technique with the help of scientific dataset Cybershake for different task sizes as 30, 50,100 and 1000. Here, Figure 4 demonstrates Power Sum Comparison of proposed COCS technique with conventional DVFS technique with the help of scientific dataset Cybershake for different task sizes as 30, 50,100 and 1000. Here, Figure 5 demonstrates Average Power Comparison of proposed COCS technique with conventional DVFS technique with the help of scientific dataset Cybershake for different task sizes as 30, 50, 100 and 1000. Similarly, Figure 6 demonstrates Power Consumption Comparison of proposed COCS technique with conventional DVFS technique with the help of scientific dataset Cybershake for different task sizes as 30, 50, 100 and 1000. Furthermore, Figure 7 demonstrates average task completion time Comparison of proposed COCS technique with various state-of-art-techniques using scientific dataset Cybershake for different task sizes as 30, 50, 100 . 
Task Completime time using proposed COCS technique

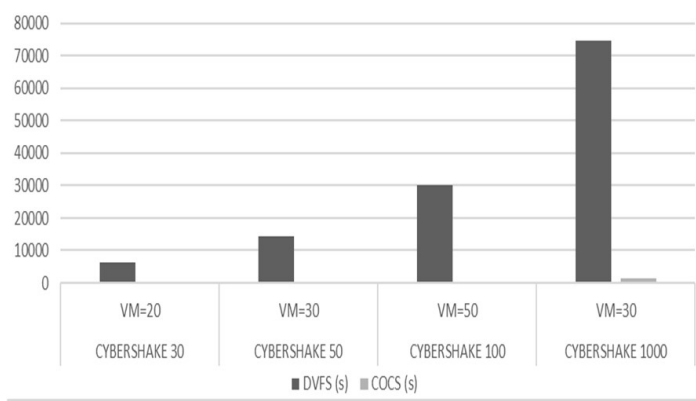

Figure 3. Task completion time comparison of proposed COCS technique vs DVFS using scientific workload Cybershake

Average Power Required comparsion using proposed COCS technique with DVFS

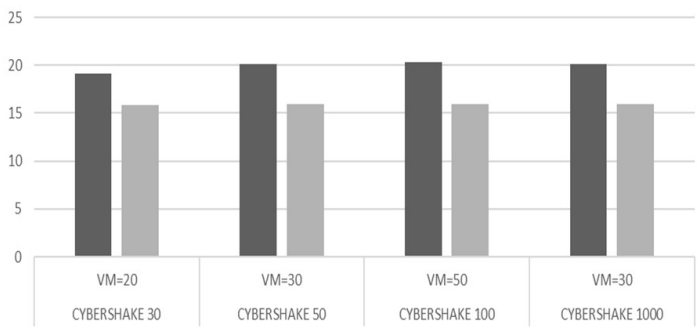

- $\operatorname{DVFS}(\mathrm{W})=\operatorname{cocs}(\mathrm{W})$

Figure 5. Average power comparison of proposed COCS technique vs DVFS using scientific workload Cybershake
Power sum comparsion using proposed COCS technique with DVFS

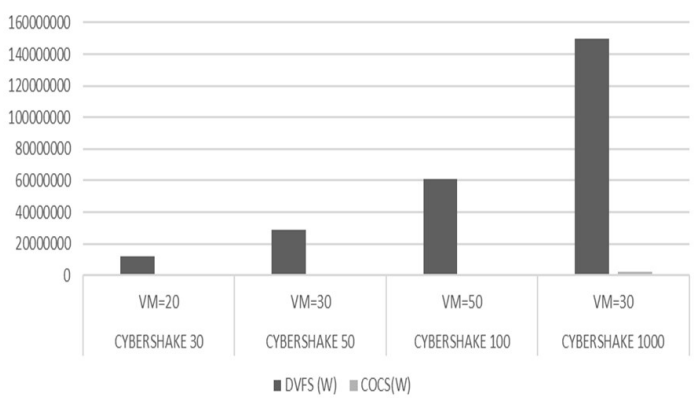

Figure 4. Power sum comparison of proposed COCS technique vs DVFS using scientific workload Cybershake

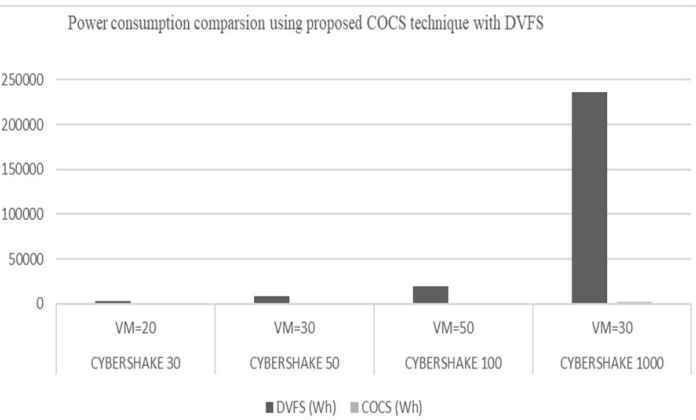

Figure 6. Power consumption comparison of proposed COCS technique vs DVFS using scientific workload Cybershake

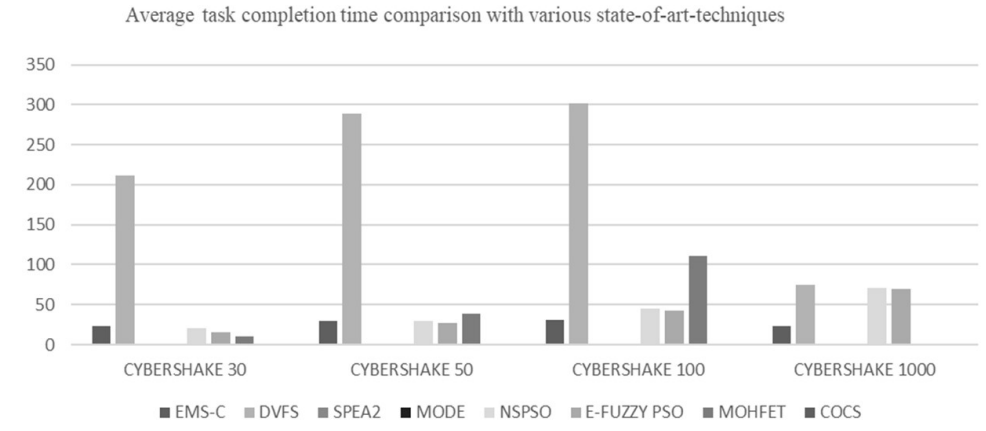

Figure 7. Average task completion time comparison of proposed COCS technique with various state-of-art-techniques using scientific workload Cybershake

\section{CONCLUSION}

An efficient task scheduling in each cloud computing VMs and reduction of high energy consumption in these devices is become a primary priority. Various researchers have provided different techniques to decrease energy consumption in multi-core cloud computing processors. However, still it remains an unsolved issue. Therefore, one method to decrease storage and energy consumption in multi-core processors is cache minimization. Therefore, we have adopted Cache Optimization Cloud Scheduling (COCS) Algorithm Based on Last Level Caches to ensure high cache memory Optimization and to enhance the processing speed of I/O subsystem in a cloud computing environment which rely upon Dynamic Voltage 
and Frequency Scaling $(D V F S)$. This technique helps to reduce last level caches and identifies the behavior of different caches in every $V M$ from different working nodes and gathers all the VMs to decrease the final cache failures. A detailed modelling is presented to minimize the energy consumption and storage in cloud computing multi-core processors by reducing cache memory. We have tested our experiment on Cybershake scientific dataset and the experimental results are compared with different conventional techniques in terms of time taken to accomplish task, power consumed in the VMs and average power required to handle tasks. The Average Task Completion Time with the proposed COCS technique for scientific dataset Cybershake 30 is $8.7576 \mathrm{sec}$, Cybershake 50 is $5.6736 \mathrm{sec}$, Cybershake 100 is 4.4321 sec and Cybershake 1000 is 1.328 sec. Power consumption using the proposed COCS technique for Cybershake 30 is 128.4845 Watts, Cybershake 50 is 151.5101 Watts Cybershake 100 is 258.672 Watts and Cybershake 1000 is 1696.490 Watts which is very less compare to any other state-of-art-techniques. Experimental results verify superiority of our proposed COCS technique in terms of task completion time, average power required and energy consumption.

\section{REFERENCES}

[1] Wikipedia, Big data, 2014a. [Online]. Available: http://en.wikipedia.org/wiki/Big_data.

[2] M. A. Beyer and D. Laney, The Importance of 'big data': A Definition. Gartner, Stamford, CT, 2012.

[3] P. Mell and T. Grance, "The NIST Definition of Cloud Computing," National Institute of Standards and Technology, 2009.

[4] H. Topcuoglu, S. Hariri, and M.y. Wu, "Performance-effective and low-complexity task scheduling for heterogeneous computing," IEEE Trans. Parallel Distrib. Syst., vol. 13(3), pp. 260-274, 2002.

[5] K. Li, "Energy-efficient task scheduling on multiple heterogeneous computers: Algorithms, analysis, and performance evaluation," IEEE Trans. Sustain. Comput., vol. 1(1), pp. 7-19, 2017.

[6] K. Li, "Power and performance management for parallel computations in clouds and data centers," J. Comput. Syst. Sci., vol. 82(2), pp. 174-190, 2016.

[7] X. Xiao, G. Xie, R. Li, and K. Li, "Minimizing schedule length of energy consumption constrained parallel applications on heterogeneous distributed systems," Proc. 14th IEEE Int. Symp. on Parallel and Distributed Processing with Applications, IEEE Computer Society, pp. 1471-1476, 2016.

[8] G. Xie, X. Xiao, R. Li, and K. Li, "Schedule length minimization of parallel applications with energy consumption constraints using heuristics on heterogeneous distributed systems," Concurrency Comput. Parctice Experience, vol. 29(16), 2016.

[9] "Enhanced intel speedstep technology for the intel pentium m processor," March 2014. [Online]. Available: http://downloa10] "Amd powernow!? technology informational white paper," November 2000. [Online]. Available: http://www.amd-k6.com/wpcontent/ uploads/2012/07/24404a.pdf

[10]K. Flautner, D. Flynn, and M. Rives, "A combined hardware software approach for low-power socs: Applying adaptive voltage scaling and intelligent energy management software," 2003. [Online]. Available: intel.com/design/network/papers/30117401.pdf

[11]V. Kelefouras, G. Keramidas, and N. Voros, "Cache Partitioning + Loop Tiling: A Methodology for Effective Shared Cache Management," 2017 IEEE Computer Society Annual Symposium on VLSI (ISVLSI), Bochum, pp. 477-482, 2017.

[12]K. Saito, R. Kobayashi, and H. Shimada, "Reduction of cache energy by switching between L1 high speed and low speed cache under application of DVFS," 2016 International Conference on Advanced Informatics: Concepts, Theory and Application (ICAICTA), pp. 1-6, 2016.

[13] M. Mavropoulos, G. Keramidas, and D. Nikolos, "A defect-aware reconfigurable cache architecture for low-Vccmin DVFS-enabled systems," 2015 Design, Automation \& Test in Europe Conference \& Exhibition (DATE), pp. 417-422, 2015.

[14] M. Kusuma, Widyawan, and R. Ferdiana, "Performance comparison of caching strategy on wordpress multisite," 2017 3rd International Conference on Science and Technology - Computer (ICST), pp. 176-181, 2017.

[15]Q. Wang and X. Chu, "GPGPU performance estimation with core and memory frequency scaling," arXiv preprint arXiv:1701.05308, 2017.

[16] S. Mittal, “A Survey of Cache Bypassing Techniques,” MDPI J. Low Power Electron. Appl., vol. 6(5), 2016.

[17] Joonho Kong and Kwangho Leeb, "A DVFS-aware cache bypassing technique for multiple clock domain mobile SoCs," IEICE Electronics Express, vol. 14(11), pp. 1-12, 2017.

[18] Y. H. Chen, Y. L. Tang, Y. Y. Liu, A. C. H. Wu, and T. Hwang, "A Novel Cache-Utilization-Based Dynamic Voltage-Frequency Scaling Mechanism for Reliability Enhancements," IEEE Transactions on Very Large-Scale Integration (VLSI) Systems, vol. 25(3), pp. 820-832, 2017.

[19]Z. Zhu, G. Zhang, M. Li, and X. Liu, "Evolutionary Multi-Objective Workflow Scheduling in Cloud," IEEE Transactions on Parallel and Distributed Systems, vol. 27(5), pp. 1344-1357, 2016.

[20]E. Deelman, D. Gannon, M. Shields, and I. Taylor, "Workflows and e-science: An overview of workflow system features and capabilities," Future Generat. Comput. Syst., vol. 25(5), pp. 528-540, 2009.

Int. J. of Adv. in Appl. Sci. Vol. 8, No. 3, September 2019: 184 - 194 